\title{
Amplitude Variations in Rotationally Split Multiplets
}

\author{
J. Robert Buchler ${ }^{1}$, Marie-Jo Goupil ${ }^{2} \&$ Thierry Serre ${ }^{1}$ \\ ${ }^{1}$ University of Florida, ${ }^{2}$ Observatoire de Paris
}

We have extended a formalism that was developed for radial pulsators (Buchler $\&$ Goupil 1984) to the nonradial ones (Goupil \& Buchler 1993). For an $\ell=1$ triplet, for example, the modal amplitudes satisfy the nonlinear amplitude equations

$$
\begin{aligned}
& \frac{d a_{-}}{d t}=\left(\kappa_{-}-i \Delta \omega_{-}\right) a_{-}+\gamma_{-} a_{+}^{*} a_{0}^{2}-2 \beta_{--} a_{-}\left|a_{-}\right|^{2}-2 \beta_{-0} a_{-}\left|a_{0}\right|^{2}-4 \beta_{-+} a_{-}\left|a_{+}\right|^{2} \\
& \frac{d a_{0}}{d t}=\kappa_{0} a_{0} \quad+2 \gamma_{0} a_{0}^{*} a_{+} a_{-}-2 \beta_{0-} a_{0}\left|a_{-}\right|^{2}-3 \beta_{00} a_{0}\left|a_{0}\right|^{2}-2 \beta_{0+} a_{0}\left|a_{+}\right|^{2} \\
& \frac{d a_{+}}{d t}=\left(\kappa_{+}+i \Delta \omega_{+}\right) a_{+}+\gamma_{+} a_{-}^{*} a_{0}^{2}-4 \beta_{+-} a_{+}\left|a_{-}\right|^{2}-2 \beta_{+0} a_{+}\left|a_{0}\right|^{2}-2 \beta_{++} a_{+}\left|a_{+}\right|^{2}
\end{aligned}
$$

The complex amplitudes $a_{m}, m=-1,0,+1$, represent the slowly varying amplitudes of the multiplet in which a phase-factor $\exp (i \omega t)$ has been removed. The asterisks denote complex conjugation. $\Delta \omega_{ \pm}$is the rotationally induced linear frequency split of the triplet. The $\kappa_{m}$ are the linear growth-rates, $\beta_{m m^{\prime}}$ and $\gamma_{m}$ are the nonlinear coupling coefficients, calculable from the stellar hydrodynamic equations, $\beta_{m m^{\prime}}$ are the usual cubic saturation coefficients, and $\gamma_{m}$ are the coupling coefficients specific to the resonance considered here, viz. $\omega_{-} \approx \omega_{0} \approx \omega_{+}$. We note that angular momentum constraints limit the types of couplings that could otherwise occur for this type of resonance.

The assumptions that underlie the derivation of these particular ampltitude equations are, first, that the stellar rotation is slow, second, that we can disregard magnetic fields, and that we can ignore coupling between multiplets.

These amplitude equations allow us to examine the nonlinear behavior of the amplitude multiplets in the Fourier spectrum in a slowly rotating, pulsating star. Large amplitude asymmetries are found to be possible in a given multiplet (which are not due to projection effects). Both steady and time-dependent amplitudes occur, depending on the coupling coefficients between the components of the multiplet.

Steady as well as modulated amplitudes can occur depending on the stellar model:

1. Steady Amplitudes, with $A_{m}=\left|a_{m}\right|$ can be of 3 types

- single nonzero amplitude (singlet, either $m=-1,0$, or +1$)$ )

- two nonzero amplitudes (doublet, $m=-1$ and $m=+1, A_{0}=0$ )

- three nonzero amplitudes (triplet, with $m=-1,0$, and $m=+1$.

Here, phase-lock causes the three peaks to be equidistant in frequency.

2. Time-Dependent Amplitudes

- periodic amplitude modulations (unsteady triplet)

- irregular amplitude modulations (intermittent triplet) 
In general, one can show that there is no 'energy conservation', i.e. the sum of the pulsation energies in the components of the multiplet is not constant. However, for some parameter values it may be approximately constant.

We stress that the nature and aspect of the solutions do not depend the scale of the growth-rates; the latter merely affect the time-scale of the amplitude modulation. However, the nature and aspect of the solutions depend sensitively on the values of the nonlinear coupling coefficients and on the relative values of the growth-rates.

Figure 1 is a typical example of the amplitudes a steady three-mode pulsation with the concomitant Fourier spectrum. The amplitudes are constant in time and phase-lock causes the Fourier peaks to be equally spaced (even when they are not in the linear case).
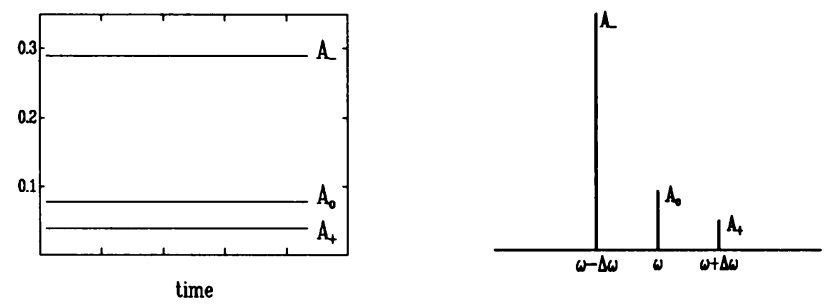

Fig. 1: Triple-mode Fixed-point, and Typical Fourier Spectrum for $\ell=1$ Multiplet

Figures 2 show examples of unsteady amplitudes. The time-dependent amplitudes (over short observing runs lead to a Fourier spectrum similar to Figure 2, but with differing results from run to run).
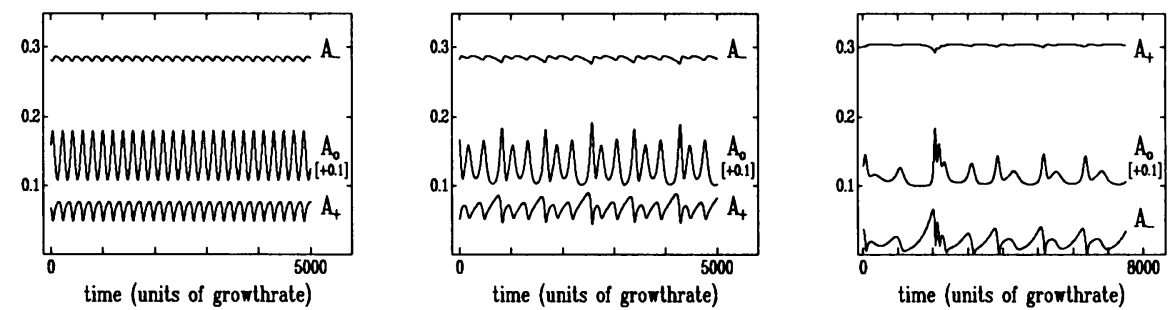

Fig. 2: Amplitudes ( $A_{0}$ shifted up by 0.1 ) left to right: periodic, irregular, intermittent

A parameter study has revealed that the amplitude equations can display a variety of different types of behavior. For example, the amplitude ratios for the different peaks are sensitive to the parameters values. Amplitude asymmetries (which are intrinsic, i.e. not due to projection effects) occur quite naturally. Ab initio computations of the nonlinear coefficients for a given stellar model, white dwarf, main sequence or other, are possible, in principle, but have not yet been carried out. Work is in progress to determine what useful constraints a comparison with observations puts on the coefficients.

References: Buchler J. R. \& Goupil M.-J. 1984, ApJ 279, 394

Goupil M.-J. \& Buchler, J. R., 1993, AA, submitted.

Work supported in part by NSF. 